\title{
DETERMINATION OF COEFFICIENTS OF CAPACITANCE OF REGIONS BOUNDED BY COLLINEAR SLITS AND OF RELATED REGIONS*
}

\author{
BY \\ BERNARD EPSTEIN \\ University of Pennsylvania
}

1. Introduction. In the study of electrostatic field problems the principal objective usually is to determine the potential and its gradient (the field strength) throughout a given domain bounded by a system of conductors. Frequently, however, it is necessary only to determine certain constants of capacitance. In this paper we consider the latter problem for a certain class of plane domains.

Let $D$ be a domain consisting of the entire plane with any finite number of slits along a single line. Several formulas for the coefficients of capacitance of such a domain are derived, two of which appear to be well suited for numerical computations. One of these formulas is based on the explicit representation of the potential as the real part of an analytic function [1] while the other formula has the feature of requiring a knowledge of the potential only on the line containing the boundary components of $D$; it does not involve any derivatives of the potential. A convenient method for determining the field along the line containing the boundary components has been given by the author in a previous paper [2].

Since the coefficients of capacitance of a domain are invariant under conformal mapping, the formulas which are derived may be employed to compute the coefficients of any domain which can be conformally mapped upon a domain $D$ of the type described above. This procedure can be applied to a certain class of domains which are of practical interest. In these cases the mapping problem involves essentially only simply-connected domains rather than multiply-connected domains. One particular case of interest, the 'bi-filar shielded cable' is considered in some detail, and as an illustration of the procedure, the coefficients of capacitance are evaluated numerically for one such domain.

2. Regions bounded by collinear slits. We consider here the problem of determining effectively the so-called coefficients of capacitance of a domain $D$ consisting of the extended $(x, y)$-plane with a finite number, $m$, of collinear slits, cut along what may be assumed to be the $x$-axis.

We recall the definition of these coefficients:

$$
p_{i k}=-\oint_{C_{k}} \frac{\partial u_{i}}{\partial n} d s, \quad(j, k=1,2, \cdots, m),
$$

where $u_{i}$, the harmonic measure of the $j$ th boundary component, is the harmonic function whose boundary values are unity on the $j$ th component and zero on all other components (the existence and uniqueness of this harmonic function is well known from the theory of the Dirichlet problem); $C_{k}$ is any curve, described in the positive sense, surrounding only the $k$ th component; and $\partial / \partial n$ indicates differentiation in the direction of the outward normal. As follows immediately from the Cauchy-Riemann equations, the $p_{i k}$ may be

\footnotetext{
*Received May 17, 1955. The research reported in this article was done at the Institute of Mathematical Sciences, New York University, and was supported by the United States Air Force, through the Office of Scientific Research of the Air Research and Development Command.
} 
defined alternatively as the increment in the harmonic function $v_{i}$ conjugate to $u_{i}$ which results when $C_{k}$ is described once in the negative sense.

We also recall several important properties of these coefficients of capacitance:

$$
\begin{gathered}
p_{i k}=p_{k j} ; \\
\sum_{k=1}^{m} p_{i k}=0, \quad j=1,2, \cdots, m ; \\
p_{i j}>0 ; \\
p_{i k}<0, \quad j \neq k .
\end{gathered}
$$

The $p_{i k}$ are, of course, conformal invariants. Hence we may assume that $D$ consists of the entire plane minus a finite number of slits lying on the $x$-axis, one of which extends to infinity in both directions (see Fig. 1); this configuration can always be realized by

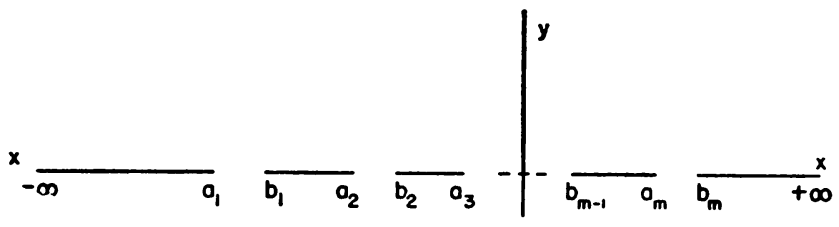

Fig. 1.

a suitable inversion. It will be seen that this assumption eliminates the possibility of any convergence difficulties. For brevity such domains will be called slit-domains. We number the finite slits $1,2, \cdots, m-1$ from left to right; and the infinite slit is the $m$ th.

We proceed to derive various formulas for the quantities $p_{i k}$ which may prove useful for numerical computations. First we shall employ the definition (2.1); later we shall derive a formula based on the alternative definition given following (2.1).

Taking into account (2.2a) and (2.2b) we see that it suffices to determine the $p_{i k}$ with $j<m, k<m$. Let $f_{j}(x)=u_{i}(x, 0)$, so that, in particular, $f_{i}(x)=0$ for $x \leq a_{1}$, $x \geq b_{m}$. By the Poisson formula we have, for any point off the $x$-axis:

$$
u_{j}(x, y)=\frac{|y|}{\pi} \int_{a_{1}}^{b_{m}} \frac{f_{i}(\xi) d \xi}{(\xi-x)^{2}+y^{2}} .
$$

We take as the curve $C_{k}$ a rectangle with vertical sides passing through the gaps $\left(a_{k}, b_{k}\right)$ and $\left(a_{k+1}, b_{k+1}\right)$. It is easily found from (2.3), by differentiating under the integral sign, that for any value of $x$ satisfying $\dot{a}_{1} \leq x \leq b_{m}$

$$
\left|\frac{\partial u_{i}}{\partial x}\right| \leq \frac{2 A^{2}}{\pi|y|^{3}}, \quad\left|\frac{\partial u_{i}}{\partial y}\right| \leq \frac{2 A}{\pi y^{2}}, \quad A=\max \left(\left|a_{1}\right|,\left|b_{m}\right|\right)
$$

(one uses, of course, the fact that $\left|f_{i}(\xi)\right| \leq 1$ ). Hence as the horizontal sides recede to infinity the contribution to the integral (2.1) from these sides vanishes, and therefore (2.1) may be written as follows:

$$
p_{i k}=I_{i, k}-I_{i, k+1},
$$

where

$$
I_{i, r}=\int_{-\infty}^{\infty} \frac{\partial u_{i}}{\partial x} d y, \quad a_{r}<x<b_{r}
$$

(of course $p_{i m}=I_{i, m}-I_{i, 1}$ ). 
We wish to rewrite $I_{i, r}$ in such a form as to involve only the values of $u_{i}$ on the $x$-axis, i.e., the values $f_{j}(x)$. If one expresses $\left(\partial u_{i}\right) / \partial x$ by differentiating (2.3), inserts this expression into (2.6), and interchanges the order of integration, one obtains the following expression for $I_{i, r}$ :

$$
I_{i, r}=\frac{2}{\pi} P \int_{a_{1}}^{b_{m}} \frac{f_{i}(\xi) d \xi}{\xi-x}, \quad a_{r}<x<b_{r} .
$$

Here the symbol $P$ denotes the Cauchy principal value of the integral.

A second expression for $I_{i, r}$ is obtained by the following artifice. Since the right side of (2.6) gives the same value for any choice of $x$ in the $r$ th gap, we integrate both sides of (2.6) over this gap. Then we obtain

$$
\left(b_{r}-a_{r}\right) I_{i .}=\int_{a_{r}}^{b_{r}} \int_{-\infty}^{\infty} \frac{\partial u_{i}}{\partial x} d y d x .
$$

Interchanging the order of integration [this is easily justified with the aid of the first inequality in (2.4)] we obtain

$$
\begin{aligned}
\left(b_{r}-a_{r}\right) I_{i, r} & =\int_{-\infty}^{\infty} \int_{a_{r}}^{b_{r}} \frac{\partial u_{i}}{\partial x} d x d y=\int_{-\infty}^{\infty}\left\{u\left(b_{r}, y\right)-u\left(a_{r}, y\right)\right\} d y \\
& =\frac{1}{\pi} \int_{-\infty}^{\infty}|y|\left\{\int_{a_{2}}^{b_{m}} f_{i}(\xi)\left[\frac{1}{\left(\xi-b_{r}\right)^{2}+y^{2}}-\frac{1}{\left(\xi-a_{r}\right)^{2}+y^{2}}\right] d \xi\right\} d y .
\end{aligned}
$$

Now we interchange the order of integration once more, and obtain the following expression for $I_{i, r}$ :

$$
I_{i, r}=\frac{2}{\pi\left(b_{r}-a_{r}\right)} \int_{a_{1}}^{b_{m}} f_{i}(\xi) \ln \left|\frac{\xi-a_{r}}{\xi-b_{r}}\right| d \xi .
$$

A third expression for $I_{j, r}$ is obtained as follows. Since $f_{i}(\xi)$ is continuous and $f_{i}^{\prime}(\xi)$ and $\ln \left|\left(\xi-a_{r}\right) /\left(\xi-b_{r}\right)\right|$ are absolutely integrable, an integration by parts enables us to rewrite $(2.9)$ in the form:

$I_{i, r}=\frac{-2}{\pi\left(b_{r}-a_{r}\right)} \int_{a_{1}}^{b_{m}} f_{i}^{\prime}(\xi)\left[\left(\xi-a_{r}\right) \ln \left|\xi-a_{r}\right|-\left(\xi-b_{r}\right) \ln \left|\xi-b_{r}\right|\right] d \xi ;$

the integrated term vanishes since $f_{i}\left(a_{1}\right)=f_{i}\left(b_{m}\right)=0$.

Equations (2.7), (2.9), (2.10), together with (2.5), give three formulas for the coefficients $p_{i k}$ which employ only the values of $u_{i}$ on the $x$-axis. These equations were all derived using the definition (2.1) of $p_{i k}$. A fourth formula is obtained by using the alternative definition, as follows. It is shown in [1], Sec. 91 that the analytic function $w_{i}(z)=u_{i}+i v_{i}$ must satisfy the condition

$$
w_{i}^{\prime}(z)=\frac{P_{i}(z)}{\left\{-\prod_{r=1}^{m}\left(z-a_{r}\right)\left(z-b_{r}\right)\right\}^{1 / 2}},
$$

where $P_{j}(z)$ is a polynomial, of degree not exceeding $m-2$, with $m-1$ real coefficients which are uniquely determined by the conditions*:

$$
\int_{a r}^{b r} w_{i}^{\prime}(z) d z=\left\{\begin{aligned}
+1, & \text { for } r=j, \\
-1, & \text { for } r=j+1 \\
0, & \text { for } r \neq j, \quad r \neq j+1 .
\end{aligned}\right.
$$

*It must be remembered that the denominator of $w_{j}(z)$ changes sign on successive intervals $\left(a_{r}, b_{r}\right)$. 
(It is shown in reference [1] that of the $m$ conditions (2.12) only $m-1$ are independent.) Now employing the alternative definition of $p_{i k}$ and taking for $C_{k}$ the doubly-counted $k$ th interval, one easily obtains from (2.11) the result

$$
p_{i k}=\mp 2 \int_{b_{k}}^{a_{k+1}} \frac{P_{i}(\xi) d \xi}{\left\{\prod_{r-1}^{m}\left(\xi-a_{r}\right)\left(\xi-b_{r}\right)\right\}^{1 / 2}},
$$

where the ambiguity in sign is most easily resolved with the aid of (2.2c) and (2.2d).

Insofar as numerical computations are concerned, it would appear that formulas (2.9) and (2.13) are especially suitable. While (2.13) has an advantage over (2.9) in that integration is necessary only over a single interval, it involves the solution of the system (2.12) for the coefficients of the polynomial $P_{i}(z)$ and this may become laborious for large values of $m$. In this case it might prove preferable to employ (2.9), obtaining the function $f_{i}(x)$ to the desired degree of approximation by the method given in [2], Sec. 6.

3. Extension to more general regions. Since the coefficients of capacitance are conformal invariants, they may be determined by the method described above for any domain which can be mapped conformally onto a slit-domain. A simple example of such a domain is the entire $(x, y)$-plane slit along a finite number of arcs of a circle (see [1], Sec. 92). By a suitable linear transformation we can map the circumference of this circle onto the $x$-axis, thus obtaining a slit-domain.

Another domain to which this method applies is a domain bounded externally by one circle and internally by two others. By a suitable linear transformation such a region may always be mapped into a domain with the centers of all three circles collinear (see Fig. 2).

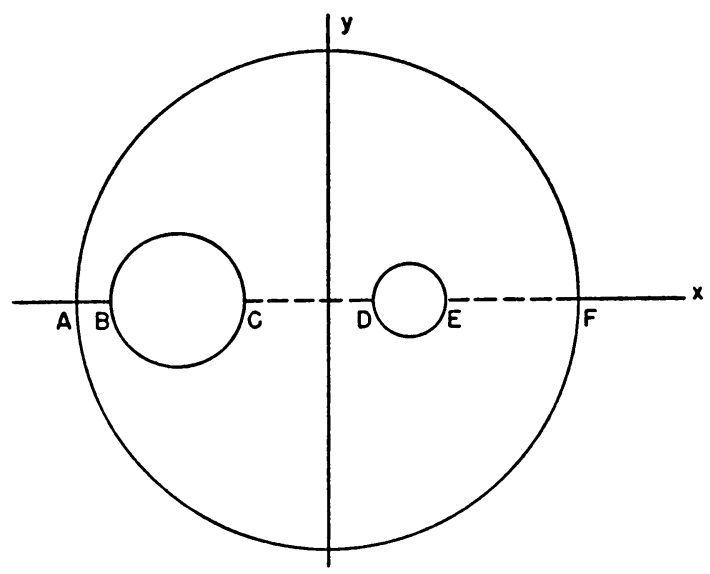

FIG. 2.

Now suppose that the upper half of this domain, which is simply connected, is mapped conformally by an analytic function $\zeta=f(z)$ upon the upper half of the $\zeta$-plane. Then the upper halves of the three circles are mapped into segments of the real axis of the $\zeta$-plane, and the three segments of the axis of symmetry $(A B, C D, E F)$ are mapped into the remainder of the real axis. By the Schwarz reflection principle, the entire domain 
of Fig. 2 is then conformally mapped onto the $\zeta$-plane with three slits along the real axis, i.e., upon a slit-domain. A particular case of practical interest in the "bi-filar shielded conductor'. This case will be discussed in some detail in Sec. 4 .

Closely related to the configuration of Fig. 2 is that of the plane with any finite number of circular apertures the centers of which all lie on one line (see Fig. 3). Each

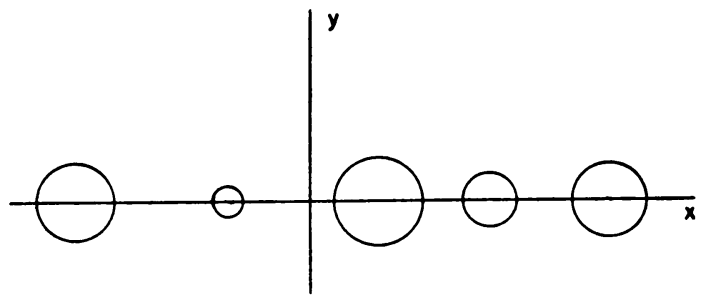

Fig. 3.

half of this region is simply connected. If, as before, we can make a conformal mapping of the upper half of this region upon the upper half of the $\zeta$-plane, then, as in the previous case, the reflection principle immediately gives the mapping of the entire configuration upon the $w$-plane with collinear slits.

The use of the Schwarz reflection principle to transform a region into one bounded by collinear slits may prove of use in studying the electrostatic field created by an electrified grid.

4. An illustrative example. In the case of the domains described in Sec. 3 it has been shown that the problem of determining the coefficients $p_{i k}$ may be reduced essentially to the problem of finding a mapping function for a simply connected domain, regardless of the connectivity of the original domain. Since the simply connected domain in question is bounded by parts of circles and straight lines, the required mapping can be obtained, in principle, by solving a certain non-linear third-order differential equation which is closely related to a certain linear second order equation (see [3], pp. 198-208). However, in practice this fact is of little use, for these differential equations contain the so-called accessory parameters of the domain, which cannot readily be determined except in the most elementary cases. But for regions. of the type under consideration, it might be possible to overcome this difficulty with the help of some of the many approximate conformal mapping techniques that have been developed.

Here, by way of an example, we shall consider a case of the 'bi-filar shielded cable' which can be treated by elementary methods.

Referring to Fig. 4, let the radius of the outer circle be taken equal to unity (this assumption does not reduce the generality), and let the points $B, C, D, E$ correspond to $z=-\beta,-\alpha, \alpha, \beta$ respectively. By the mapping function

$$
\zeta=(\alpha \beta)^{1 / 2}\left(z+\frac{1}{z}\right)
$$

the interior of the outer circle is mapped upon the exterior of the slit $-2(\alpha \beta)^{1 / 2} \leq$ $\zeta \leq 2(\alpha \beta)^{1 / 2}$, and hence the domain bounded by the three circles is mapped onto the $\zeta$-plane minus the aforementioned slit and the images of the two interior circles. In particular, if $\beta \ll 1$, so that the inner circles are small and located close to the center of 
the outer circle, then the mapping (4.1) may be approximated in the neighborhood of the inner circles by the linear mapping

$$
\zeta=\frac{(\alpha \beta)^{1 / 2}}{z}
$$

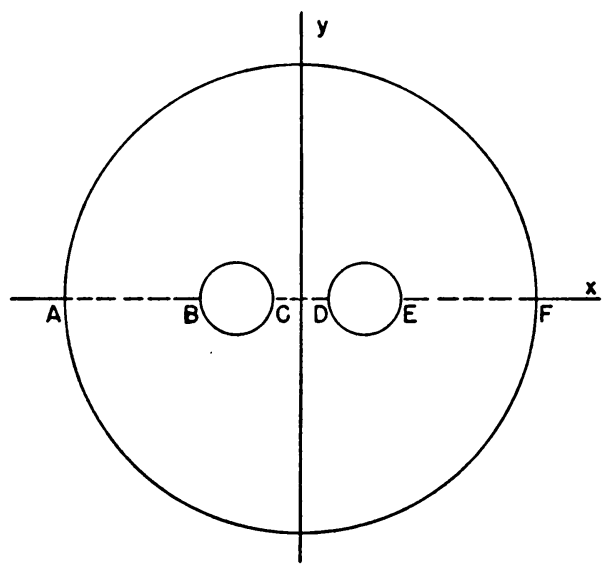

FIG. 4.

which transforms the inner circles into circles which meet the real axis at $\pm(\alpha / \beta)^{1 / 2}$ and $\pm(\beta / \alpha)^{1 / 2}$. Hence the domain of Fig. 4 is mapped by (4.1) approximately into the region indicated in Fig. 5. The centers of the circles in Fig. 5 are given by $\zeta= \pm a$,

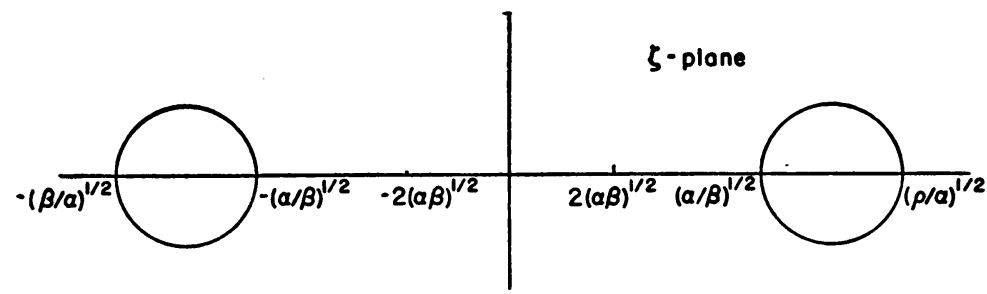

Fra. 5.

where $a=(\alpha+\beta) /\left[2(\alpha \beta)^{1 / 2}\right]$, and the radius of each circle is given by $R=(\beta-\alpha) /$ $\left[2(\alpha \beta)^{1 / 2}\right]$. Since $a^{2}-R^{2}=1$, the exterior of these two circles may be mapped upon the exterior of two slits of the real axis by the function (see [3], p. 297, example 9)

$$
w=\frac{1+f[(1+\zeta) /(1-\zeta), \rho]}{1-f[(1+\zeta) /(1-\zeta), \rho]},
$$

where

and

$$
\rho=\frac{(\beta)^{1 / 2}-(\alpha)^{1 / 2}}{(\beta)^{1 / 2}+(\alpha)^{1 / 2}}
$$

$$
f(t, \rho)=\frac{\rho^{2}}{t} \frac{\prod_{n=0}^{\infty}\left(1+\rho^{8 n-2} t^{2}\right) \prod_{n=1}^{\infty}\left(1+\rho^{8 n+2} t^{-2}\right)}{\prod_{n=0}^{\infty}\left(1+\rho^{8 n+2} t^{2}\right) \prod_{n=0}^{\infty}\left(1+\rho^{8 n+8} t^{-2}\right)}
$$


(see [3], Chap. VI, Sec. 3, Eqs. (32) and (49)). The circles map onto the twice-counted slits

$$
-\frac{1+L(\rho)}{1-L(\rho)} \leq w \leq-\frac{1-L(\rho)}{1+L(\rho)}
$$

and

$$
\frac{1-L(\rho)}{1+L(\rho)} \leq w \leq \frac{1+L(\rho)}{1-L(\rho)}
$$

respectively, where

$$
L(\rho)=2 \rho \prod_{n=1}^{\infty}\left(\frac{1+\rho^{8 n}}{1+\rho^{8 n-4}}\right)^{2} .
$$

The interval $-2(\alpha \beta)^{1 / 2} \leq \zeta \leq 2(\alpha \beta)^{1 / 2}$ maps onto the infinite interval

$$
|w| \geq \frac{1-f\left[\left\{1+2(\alpha \beta)^{1 / 2}\right\} /\left\{1-2(\alpha \beta)^{1 / 2}\right\}, \rho\right]}{\left.\left.1+f\left[\left\{1+2(\alpha \beta)^{1 / 2}\right\} /\right\} 1-2(\alpha \beta)^{1 / 2}\right\}, \rho\right]}
$$

of the real axis. Thus the domain of Fig. 4 has been approximately mapped into a slitdomain, and the formulas given there may be employed to determine the $p_{i k}$.

To give a numerical illustration, let $\alpha=1 / 20, \beta=1 / 5$. Then $\rho=1 / 3$, and, since the infinite products (4.4) and (4.5) converge rapidly, one obtains very easily

$$
\begin{gathered}
\frac{1-L(\rho)}{1+L(\rho)}=0.212, \quad \frac{1+L(\rho)}{1-L(\rho)}=4.726, \\
\frac{1-f\left[\left\{1+2(\alpha \beta)^{1 / 2}\right\} /\left\{1-2(\alpha \beta)^{1 / 2}\right\}, \rho\right]}{1+f\left[\left\{1+2(\alpha \beta)^{1 / 2}\right\} /\left\{1-2(\alpha \beta)^{1 / 2}\right\}, \rho\right]}=8.628
\end{gathered}
$$

In order to work with more convenient numbers, we employ the transformation

$$
Z=\frac{.2}{.212} Z,
$$

thus obtaining in the $Z$-plane a slit-domain characterized by the following numbers (see Fig. 1):

$a_{1}=-8.1, \quad b_{1}=-4.5, \quad a_{2}=-0.2, \quad b_{2}=0.2, \quad a_{3}=4.5, \quad b_{3}=8.1$.

On account of the low connectivity of the domain $(m=3)$ it was decided to employ formula (2.13) to evaluate the $p_{i k}$. The coefficients $p_{22}$ and $p_{21}$ (the numbering of the boundary components is given in Fig. 4 and Fig. 5 , and is in accordance with the numbering given preceding Fig. 1) were determined as follows. The polynomial $P_{2}(Z)$, which according to the discussion of Sec. 2 is of first degree, was replaced in (2.12) by the quadratic polynomial $a Z^{2}+b Z+c$ with unknown coefficients $a, b, c$. The integrals appearing in (2.12) were computed by interpolation (the Tchebyshev five-point formula was used) and the resulting system of linear equations was solved for the coefficients $a, b, c$, with the following results:

$$
a=-9.3 \times 10^{-8}, \quad b=-1.962, \quad c=-11.608 .
$$


(The radical appearing in (2.12) was taken positive on the first and third intervals and negative on the second interval.) The extremely small value obtained for $a$, whose exact value must be zero, serves as an excellent check on the accuracy of the computations. The expression for $P_{2}(Z)$ thus obtained was then employed in (2.13), with the following results:

$$
p_{21}=-2.153, \quad p_{22}=+3.820 .
$$

By symmetry, $p_{11}=p_{22}$, and now the remaining six coefficients are obtained with the aid of (2.2a) and (2.2b). Thus, the following table of values of the $p_{i k}$ was obtained.

\begin{tabular}{|c|c|c|c|}
\hline & 1 & 2 & 3 \\
\hline 1 & +3.820 & -2.153 & -1.667 \\
\hline & -2.153 & +3.820 & -1.667 \\
\hline & -1.667 & -1.667 & +3.334 \\
\hline
\end{tabular}

As a check, $p_{22}$ was also computed by means of (2.5) and (2.10), and excellent agreement was obtained.

Acknowledgement. We wish to thank Mr. Charles Kahane, who so capably carried out the computations connected with the illustrative example of Sec. 4.

\section{REFERENCES}

[1] N. I. Muskhelishvili, Singular integral equations, P. Noordhoff Ltd

[2] B. Epstein, Quart. Appl. Math. 6, No. 3, 301-317 (Oct. 1948)

[3] Z. Nehari, Conformal mapping, McGraw-Hill 